\title{
Comparing motivational, self-regulatory and habitual processes in a computer- tailored physical activity intervention in hospital employees - protocol for the PATHS randomised controlled trial
}

Dominika Kwasnicka ${ }^{1,2}$, Corneel Vandelanotte ${ }^{1,2^{*}}$ (D), Amanda Rebar ${ }^{1,2}$, Benjamin Gardner ${ }^{3}$, Camille Short ${ }^{4}$, Mitch Duncan ${ }^{5}$, Dawn Crook ${ }^{6}$ and Martin S. Hagger ${ }^{1,2,7}$

\begin{abstract}
Background: Most people do not engage in sufficient physical activity to confer health benefits and to reduce risk of chronic disease. Healthcare professionals frequently provide guidance on physical activity, but often do not meet guideline levels of physical activity themselves. The main objective of this study is to develop and test the efficacy of a tailored intervention to increase healthcare professionals' physical activity participation and quality of life, and to reduce work-related stress and absenteeism. This is the first study to compare the additive effects of three forms of a tailored intervention using different techniques from behavioural theory, which differ according to their focus on motivational, self-regulatory and/or habitual processes.

Methods/Design: Healthcare professionals ( $N=192)$ will be recruited from four hospitals in Perth, Western Australia, via email lists, leaflets, and posters to participate in the four group randomised controlled trial. Participants will be randomised to one of four conditions: (1) education only (non-tailored information only), (2) education plus intervention components to enhance motivation, (3) education plus components to enhance motivation and self-regulation, and (4) education plus components to enhance motivation, self-regulation and habit formation. All intervention groups will receive a computer-tailored intervention administered via a web-based platform and will receive supporting text-messages containing tailored information, prompts and feedback relevant to each condition. All outcomes will be assessed at baseline, and at 3-month follow-up. The primary outcome assessed in this study is physical activity measured using activity monitors. Secondary outcomes include: quality of life, stress, anxiety, sleep, and absenteeism. Website engagement, retention, preferences and intervention fidelity will also be evaluated as well as potential mediators and moderators of intervention effect.

(Continued on next page)
\end{abstract}

\footnotetext{
* Correspondence: c.vandelanotte@cqu.edu.au

${ }^{1}$ Health Psychology \& Behavioural Medicine, School of Psychology and Speech Pathology, Faculty of Health Sciences, Curtin University, Perth, Australia

${ }^{2}$ Physical Activity Research Group, School of Health, Medical and Applied Sciences, Central Queensland University, Rockhampton, QLD, Australia

Full list of author information is available at the end of the article
} 
(Continued from previous page)

Discussion: This is the first study to examine a tailored, technology-supported intervention aiming to increase physical activity in healthcare professionals. The study will evaluate whether including additional theory-based behaviour change techniques aimed at promoting motivation, self-regulation and habit will lead to increased physical activity participation relative to information alone. The online platform developed in this study has potential to deliver efficient, scalable and personally-relevant intervention that can be translated to other occupational settings.

Trial registration: Australian New-Zealand Clinical Trial Registry: ACTRN12616000462482, submitted 29/03/2016, prospectively registered 8/04/2016.

Keywords: Computer-tailoring, Behaviour change, Behaviour maintenance, Habit, Healthcare professionals, Physical activity, Web-based, Randomised controlled trial

\section{Background}

Physical inactivity is related to increased risk of a number of chronic diseases (e.g., cardiovascular disease, Type 2 diabetes, certain types of cancer, obesity) [1, 2]. Engaging in regular physical activity is related to reduced risk of chronic diseases [2]. In addition, positive outcomes of physical activity include improved quality of life, better sleep, and reduced stress [3, 4]. World Health Organisation physical activity guidelines for adults to gain health benefits is to undertake at least $150 \mathrm{~min}$ of moderate intensity physical activity per week (e.g., walking, cycling), or at least 75 min of vigorous intensity physical activity per week (e.g., running, playing football) [5]. Globally, 1 in 4 adults does not meet these recommendations [5].

Healthcare professionals have a key role to play in the promotion of physical activity [6]; however they often do not meet guideline levels of physical activity themselves. For instance a study focussing on nurses' physical activity $(N=325)$ indicated that more than half of the assessed sample did not meet guideline levels of physical activity [7]. Those who were less active were also more likely to report poor general health and worse sleep patterns than their active counterparts. Despite significant health education among health care professionals, it appears that the health knowledge often does not translate into their own health behaviours [7-9].

In addition, there is a relationship between personal physical activity behaviours of healthcare professionals and their health-promotion practice. A systematic review [10] of cross-sectional studies $(N=13)$ investigating this relationship found that a higher personal physical activity level in healthcare professionals was associated with higher physical activity-promoting practices in most studies. Health professionals with positive attitudes towards physical activity were also more likely to promote physical activity to their patients.

Healthcare professionals often exhibit unhealthy lifestyle behaviours with work-related stress identified as the most frequently-cited reason [11]. Professionals report that hospitals are a highly stressful work environment, and irregular shift work often places an additional strain on the hospital employees [12]. Sleep patterns and sleep quality among healthcare professionals were insufficient for good health [13] and physical activity is a recognised means to improve sleep quality [14]. In addition, nurses are prone to suffer lower back pain [15] and physical activity is recommended to this occupational group for managing back pain. As such, there is strong rationale for promoting physical activity among healthcare professionals.

The workplace is reported as a suitable environment for making changes in the physical activity and improving health of employees [16]. Increased participation in physical activity in healthcare professionals can be promoted using behavioural interventions which utilise persuasive strategies and techniques to encourage individuals to change their behaviour (e.g., goal setting, planning, providing social support) $[17,18]$. Such interventions have been shown to be effective in promoting increased uptake and maintenance of physical activity, including in the workplace [19-21], hence they may be suitable for promoting physical activity to healthcare professionals.

Other than identifying the importance of context in the delivery of interventions, such as the occupational setting for healthcare workers, it is also important to look at the means by which interventions may be delivered to employees in this context [22]. Recently computer-tailoring has been used to deliver behavioural interventions in health contexts and it has received increased attention as a means to effectively deliver personalised interventions to a wide audience at a relatively low cost [23]. These interventions provide users with individualised feedback based on their demographic profile and preferences. Users are prompted to provide information salient to the intervention (using an online questionnaire) and are subsequently provided with tailored feedback including information on behaviour change matched to their requirements and consistent with their responses. Pre-defined algorithms generate tailored content for the user based on userprovided information and the relevant behaviour change content that is stored in a database including all possible response options [24]. 
Web-based interventions have a number of advantages over face-to-face interventions: they have wide reach, comparatively low cost of implementation and delivery, and flexibility of intervention use at times and location convenient for the user $[25,26]$. Tailoring web-based interventions also carries distinct advantages over nontailored approaches: participants are presented only with relevant and personalised information, and non-relevant information can be omitted. This in turn may increase engagement and persuasion [27, 28]. In computertailored interventions, less information is presented to the users and more attention is directed to the relevant intervention content. In addition, consistent with the elaboration likelihood model of persuasion [29], information in the intervention is more likely to be thoughtfully attended to when it is personally relevant and when readers are motivated. Therefore, tailored interventions are more likely to be attended to and should lead to longer lasting behavioural change.

In addition to computer tailoring, there is also a growing literature supporting the use of short message service (SMS) or 'text' messaging to promote health behaviour. Research has demonstrated that delivering health behaviour messages via text messaging can promote health behaviour change in numerous contexts, including physical activity [30]. Text messaging has also been used in conjunction with web-based interventions to improve health behaviour and to enhance their effectiveness. A systematic review reported that the effectiveness of webbased interventions can be enhanced by the inclusion of SMS as a means to remind participants of intervention content or to augment it [31]. Therefore, adoption of webbased tailored interventions to promote physical activity augmented with text messaging, may be a useful, costeffective means to promote physical activity to healthcare professionals.

In the context of physical activity promotion, computer-tailored behavioural interventions have been reported as effective [26, 32]; however further investigation of the mechanisms is needed in terms of evaluating these interventions and their effectiveness across multiple contexts. Investigating the mechanisms that can improve the effectiveness of computer-tailored interventions is crucial. Therefore, studies that isolate specific behaviour change techniques (BCTs) within intervention conditions using factorial designs will provide robust evidence for testing the impact of specific BCTs [18]. This means that knowledge of the specific individual components that are most likely to change behaviour will be identified.

\section{Theories of behaviour change and maintenance}

Health behaviour change interventions are usually underpinned by psychological theories of behaviour change and maintenance. Many such theories have focused on motivation and intentions to explain and change behaviour (e.g., the Theory of Planned Behaviour) [33]. These theories view lack of engagement in health behaviours (e.g., physical activity) as primarily a problem with motivation, such that increasing motivation will directly lead to increases in behavioural participation. The theories also assume that motivation is a function of an individuals' explicit, consciously-held beliefs about the behaviour [33]. However, theories of volition [34] indicate that motivation is a necessary but insufficient condition for behavioural enactment and suggests that volitional strategies (e.g., planning) that operate in a post-decisional manner (i.e. after intentions have been formed) lead to the effective execution of intentions into action because they enable an individual to more effectively recall their intended behaviour via prompts or cues that have been linked to the desired behaviour [35].

Dual process theories offer a broader perspective that integrates motivational and volitional processes, but also recognises that behaviour may also be influenced by more implicit, non-conscious processes [36-39]. Dual process theories propose that health behaviours are enacted through two processes - the conscious, deliberative processes such as those described in motivational and volitional theories, as well as non-conscious processes that occur automatically, outside an individual's deliberation. The implicit processes reflect initiating or engaging in actions with very little deliberative, reasoned, and conscious decision making processes outlined in traditional motivational theories. Behaviours that may initially be controlled through deliberative, motivational pathways behaviours can, through a habitformation process become controlled by automatic processes [40]. Habit formation likely occurs through learning of context-behaviour associations via contextdependent repetition of the behaviour usually in the presence of some rewarding contingency [41]. In some cases, the behaviour is enacted because repetition and experience has led the behaviour to occur without the need for any conscious control, as such the behaviour is classed as 'habitual' [42, 43].

Researchers applying these theories to predict and change behaviour have identified strategies and techniques to promote the development of habits [40]. These techniques should be separable and able to be isolated from techniques that promote, for example, increased behavioural engagement via the motivational and volitional pathways identified in dual process theories. As a consequence, factorial-type designs should be able to demonstrate the unique effects of techniques related to motivation and volition and techniques related to habit promotion on health behavior change. Such designs may also permit demonstration of whether the addition of 
components targeting specific processes (e.g., motivational, volitional, habitual) may lead to incremental changes in behaviour relative to each set of techniques alone.

\section{The present study}

There is a need for cost-effective, efficacious behavioural interventions to increase physical activity in healthcare professionals [13]. Research has suggested that theorybased interventions adopting multiple BCTs are effective in increasing physical activity behaviour [31]. Recent research efforts have focused on linking BCTs with underlying theoretical mechanisms of action [44]. However, few studies have systematically examined the efficacy of specific groups of BCTs that are thought to operate through one of the three specified processes (motivational, volitional, and habit-forming) and assessed their independent and additive effects on behaviour change. The purpose of the current study, the Physical Activity Tailored intervention in Hospital Staff (PATHS) study, is to evaluate the efficacy of a computer-tailored intervention to increase physical activity and quality of life, and decrease work-related stress and absenteeism, in hospital healthcare workers. The intervention will examine the additive effects of groups of BCTs derived from theories of motivation, volition, and habit on physical activity behavior change. The research is unique as it will provide the first evidence of the efficacy of techniques derived from these three theoretical approaches applied in a workplace context. The study will adopt a randomised controlled design to test and compare the effects of three distinct groups of BCTs derived from theory and focusing different processes of behaviour changes on study outcomes: motivation, self-regulation, and habit formation.

Specifically, the intervention will include motivational techniques to increase motivation and self-efficacy to engage in physical activity, self-regulatory techniques to increase capability and skills to initiate and regulate behaviour change, and habit-based techniques to increase the automatic, non-conscious tendencies to engage in physical activity on presentation of contextual and timerelevant cues. Three intervention conditions are proposed, each defined by the sets of BCTs in an additive design: (1) motivational BCTs; (2) motivational plus self-regulatory BCTs; and (3) motivational plus self-regulatory and habit formation BCTs. The intervention groups will be compared to a control condition that will receive basic non-tailored information about physical activity.

\section{Methods}

\section{Participants}

Full- and part-time hospital staff including midwives, clinical nurse managers, clinical nurses, registered nurses, enrolled nurses, and patient care assistants will be eligible to participate in the PATHS study. Participants will be recruited from hospitals in Perth, Western Australia. Staff working in shift and non-shift patterns will be eligible to participate. No restrictions will be placed on the type of contract and number of hours worked. Participants are eligible if they fail to meet the recommended weekly level of 150 min of moderate intensity physical activity because the intervention is designed for people who fail to meet this level.

Individuals who self-report a physical condition or impairment preventing them from being physically active will be excluded from the study. They will be screened using the Physical Activity Readiness Questionnaire (PAR-Q) prior to study consent [45]. However, individuals who are not eligible to participate in the study based on PAR-Q measures can still consult their GP to obtain approval. Hospital employees who do not have a mobile phone that allows them to receive text messages, who do not have access to internet on their mobile phone, computer or tablet outside of work, who are already meeting weekly recommended levels of physical activity or are currently participating in another physical activity program (e.g., a structured weight loss program, regular meetings with personal trainer or coach) will be excluded.

\section{Recruitment}

Participants will be recruited from different hospital wards via email messages and printed letters that will be sent to all potentially eligible employees, accompanied by a description of the study and its requirements and inviting them to take part. Study posters and leaflets advertising the study will be placed throughout hospital premises. Members of the PATHS team will also visit each ward to promote the study, alongside ward managers. The promotional materials will advertise the study as research investigating the development of a web-based tool to improve employees' physical activity, wellbeing and health. Recruitment materials (including posters, leaflets, emails, and printed letters) will direct interested staff to the project website, which will provide study information and will allow them to assess eligibility and register to participate. A formal statement of consent will be signed in person at the first meeting with the study researcher.

\section{Study design}

The PATHS study is a pragmatic four-group intervention trial adopting a randomised control design with assessments of primary and secondary outcomes at baseline and 3-month follow-up time points (see Fig. 1). We chose randomisation of individuals within hospitals (rather than randomisation of hospitals or wards) because individual randomisation was more efficient and more feasible with continuous recruitment of the participants; program was delivered to individuals rather than groups 


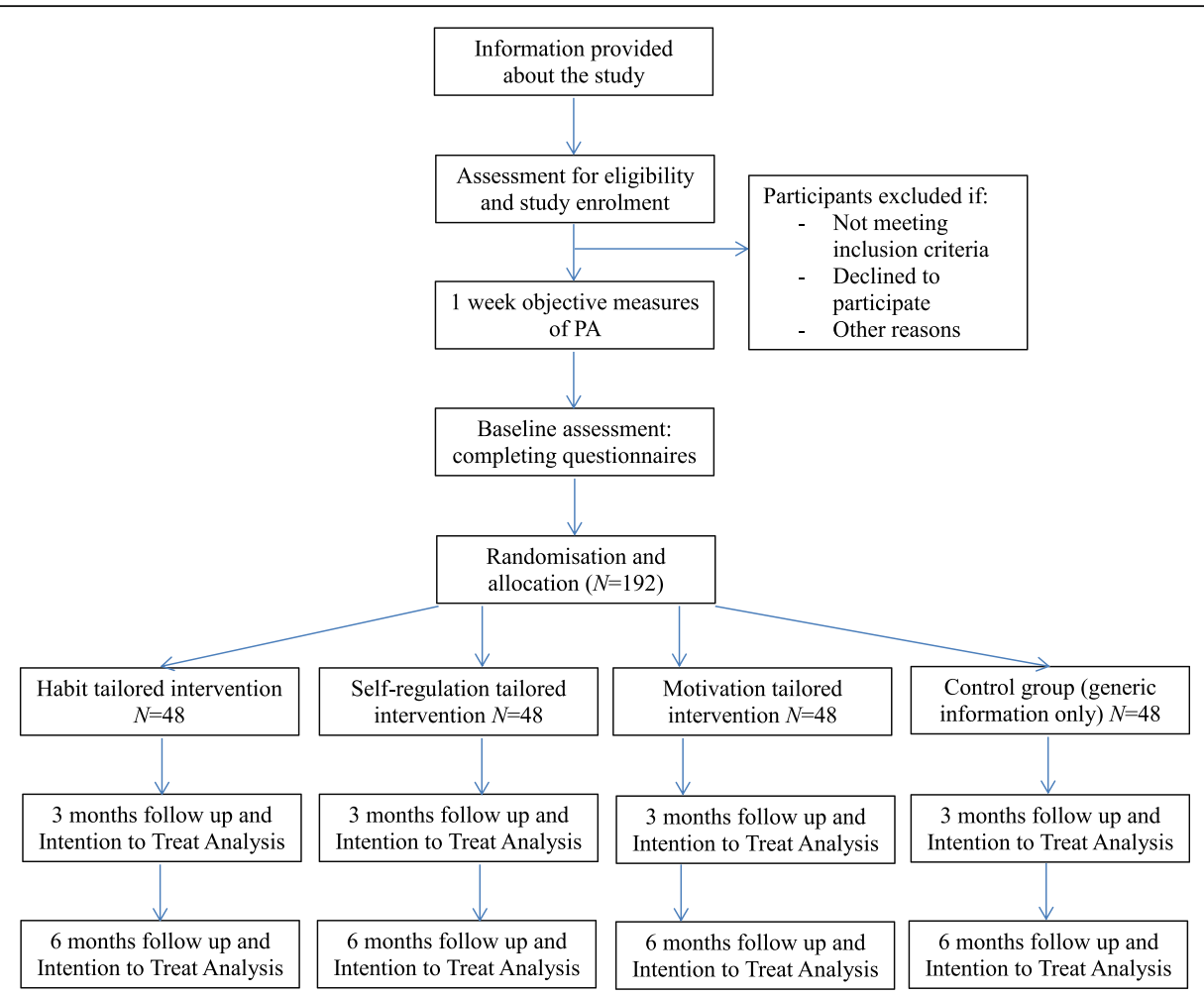

Fig. 1 CONSORT flow diagram [91]

so contamination across intervention groups was not considered a major risk [46]. The project comprises three intervention conditions and a no intervention condition, in which participants receive information about the study only. This will serve as a control condition. Each condition includes a group of theory-based BCTs designed to test the additive effects of the specific BCT groups on study outcomes:

1. Control condition - provision of information about physical activity only

2. Motivation condition - provision of information + motivational BCTs

3. Self-regulation condition - provision of information + motivational BCTs + self-regulatory BCTs

4. Habit condition - provision of information + motivational BCTs + self-regulatory BCTs + habit formation BCTs

\section{Procedure}

After screening, eligible participants will be contacted by a study researcher who will arrange a one-on-one meeting (Figure 1, CONSORT flow diagram). During the meeting, the participant will receive a detailed explanation of the study, and its requirements and expectations from the researcher. They will also be provided with an information sheet providing the same information and outlining their rights to confidentiality and to withdraw from the study at any stage without consequence. Once the participant has had the opportunity to confirm their understanding of the project and have any questions answered, they will be asked to sign an informed consent form.

A study researcher will set up and initiate a GENEActiv activity monitor [47] for each participant, including programming the participant's code number, date of birth, height, and handedness. Each monitor will be programmed to start collecting data from set up. Study participants will be required to wear the monitor on their non-dominant upper arm and will be provided with detailed instructions on how to wear the device. Participants will be instructed to wear it continuously throughout day and night for one week after the first meeting.

Eight days later, participants will meet with the researcher again to return the activity monitor. The study facilitator will check if sufficient data have been collected by the device (i.e., at least five days of wear with a minimum $16 \mathrm{~h}$ wear per day) [48]. If the participant fails to wear the activity monitor for the minimum time specified, they will be asked to wear it again for another seven days. At this second meeting, participants will 
also be asked to complete web-based baseline questionnaires using a provided tablet (Time 1).

After the second meeting, participants will be emailed a personalised login/password, and link to the website so they are able to login to the webbased PATHS study platform used to deliver the tailored intervention messages. Participants will be automatically randomly allocated online in an equal ratio to one of the four possible study conditions; a block randomisation will be used with block size of 16. If allocated to one of the three intervention conditions, they will be able to complete the first intervention session on the website immediately after completing the baseline assessments. If allocated to the control condition, they will receive an email directing them to the Library site for physical activity relevant information. The Library is a sub-section of the website with non-tailored evidence-based information about physical activity,

Across all conditions, participants will be encouraged to use and interact with the web-based PATHS platform for three consecutive months, completing sessions every fortnight. At the end of month three, participants will meet with the researcher and will be provided with a GENEActiv activity monitor to be worn for one week immediately post-intervention to assess physical activity. Participants will meet with the researcher again one week after they received the activity monitor to complete the post-intervention questionnaires and return the activity monitor (Time 2). All researchers collecting the data will be blinded to the allocation of participants' study condition.

Following the three-month period, participants will be thanked for their study contribution via personal email and text message, and their names will be automatically entered into a prize draw allowing them to win shopping vouchers for their participation (four shopping vouchers, 20 AUD value per each hospital). Participants allocated to the control condition will then be given the opportunity to receive the content from one of the intervention conditions so that they are not prevented from participating in a potentially effective intervention. They will receive login details, password and link to the web-based tailored platform providing them with the option to engage in one of the three intervention conditions, chosen randomly. All study participants will be told that there are four different versions of the same program that differ in content and behaviour change techniques used. Participants will be asked not to share or compare intervention content to avoid study contamination among participants working on the same ward. They will be asked in the post intervention evaluation if they adhered to the aforementioned recommendation.

\section{Intervention content Control condition}

Participants assigned to the control condition will be provided with information about physical activity recommendations compiled in the Library and will have the opportunity to rate the content (ranging from 0 stars [not interesting and not useful] to 5 stars [very interesting and very useful]). The presentation order of the articles will move up or down on the list based on the ratings given by the platform users, with the ones rated the highest displayed at the top of the page. The information will be factual and non-personalised. BCTs used in the control group are: providing information about health consequences [49], providing information about social [50], environmental [51] and emotional consequences [52]. Websites that provide information only have not been shown to have strong effects on health behaviour change [27] and is effective as a control group in the current study as it provides a control for information load and contact with the study team.

\section{Motivation condition}

Participants assigned to the motivation condition will receive personalised messages based on $\mathrm{BCT}$ s to promote intention and self-efficacy to engage in physical activity [53]. Specifically, participants will be provided with advice on how to work towards their goals and to maintain motivation in the face of barriers $[54,55]$.

\section{Self-regulation condition}

Participants assigned to the self-regulation condition will receive the content from the motivation condition along with content promoting self-regulation. Specifically, participants will be encouraged to set outcome-specific goals [56] and to self-monitor their progress towards these goals by monitoring how much time they spent doing physical activity. They will also be prompted to form action and coping plans [57], specifying when, where and how they will perform their physical activities, and identifying barriers to their physical activity participation and how they might overcome them. Participants will be encouraged to specifically focus on self-monitoring in high risk situations [58], such as when they are tired or stressed and their capacity for selfregulation is most likely to be compromised [59, 60]. Participants will also receive information on managing multiple goals [61] and self-regulating the situation when competing activities arise (e.g., the desire to be active and healthy conflicts with other situational options such as watching TV and resting).

\section{Habit condition}

Participants assigned to the habit condition will receive the content from the motivation and self-regulation 
conditions along with content promoting habit development (e.g., recognising prompts to action and learning to act upon these prompts). Participants will receive information relevant to implementation intentions with contextspecific prompts [62] (e.g., forming simple promptdependent plans to engage in physical activity when the prompt occurs). They will be asked to recognise and identify situational, contextual and time-based cues that can prompt them to be more active. Participants will be encouraged to develop a habit of instigating physical activity without having to consciously remember [63]. In order to do things automatically behavioural repetition of the same activity in the same contexts will be encouraged; participants will have the choice of which context they wish to do the activity.

\section{Intervention delivery}

The intervention will be delivered via the PATHS website [64] (see Fig. 2 for screenshot examples). Participants in all conditions will log-in to the web-based intervention using their email address and password created while going through the eligibility assessment process. Participants in all conditions will have access to the Library in the form of brief clear-language descriptions of physical activity recommendations and information on the importance of physical activity. Given research demonstrating that providing information about health behaviour alone is not sufficient to change behaviour [65], but also recognising the need to provide a rationale for engaging in the website and to ensure that information is controlled across conditions, all study participants will have access to the Library.

All participants will also have access to Frequently Asked Questions page, a sub-section of the website about practicalities of its use (e.g., how to change their password, basic features, tips on navigating through the website). This sub-section of the website has been designed based on piloting previous similar website [66]. Participants will be able to contact an intervention facilitator if they have any additional questions about participating in the study. There will also be an About page available - a sub-section of the website that will (1) explain the reasons why the intervention was developed and (2) introduce the PATHS study research team to add credibility. A Frequently Asked Questions page and an About page, also developed from previous investigations piloting the platform, will be slightly different for each intervention condition to account for the differences between conditions.

Participants allocated to the three intervention groups will have access to additional intervention features. Each fortnight, they will be prompted via email to access the website and to answer short online surveys and receive tailored feedback based on the information provided in their responses. Depending on their group allocation (Tables 1 and 2), participants will receive feedback aimed at promoting motivation (motivation group), motivation and self-regulation (self-regulation group), or motivation, self-regulation and prompts for actions (habit group). Information pertinent to the participant's condition will be delivered via personally-tailored messages presented as text on a webpage accompanied by appropriate pictures and graphs with tailored information (e.g., graphs showing participant's change in physical activity or weight throughout the intervention). The intervention will include six consecutive sessions that will be presented fortnightly for three consecutive months.

Each fortnight, participants will be provided with a new session including a recap of the messages from the previous two weeks along with new personally-relevant content and tailored feedback. If participants do not complete the session on time, they will be able to do so when they next login. Each session will end with a pop-up

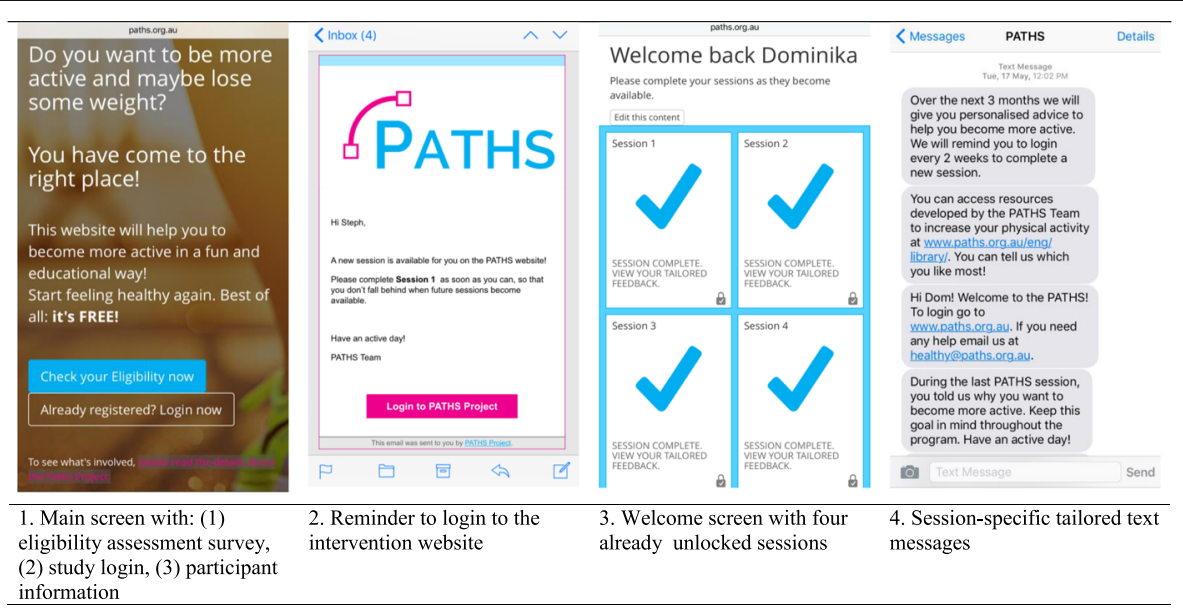

Fig. 2 PATHS study - intervention screenshots - content examples 
Table 1 Intervention content for PATHS study - overview

\begin{tabular}{|c|c|c|c|}
\hline Session number & Motivation condition & Motivation + self-regulation condition & Motivation + self-regulation + habit condition \\
\hline 1 & Motivation and self-efficacy & $\begin{array}{l}\text { Motivation, self-efficacy and } \\
\text { self-monitoring }\end{array}$ & $\begin{array}{l}\text { Motivation, self-efficacy, self-monitoring } \\
\text { and habit development }\end{array}$ \\
\hline 2 & Goal setting & Goal setting and action planning & $\begin{array}{l}\text { Goal setting, action planning and forming } \\
\text { positive habits }\end{array}$ \\
\hline 3 & $\begin{array}{l}\text { Self-efficacy, barriers identification } \\
\text { and staying motivated when } \\
\text { facing barriers }\end{array}$ & $\begin{array}{l}\text { Self-efficacy, barrier identification } \\
\text { and staying motivated and } \\
\text { self-regulating when facing barriers }\end{array}$ & $\begin{array}{l}\text { Self-efficacy, barriers identification and staying } \\
\text { motivated, self-regulating in face of barriers and } \\
\text { recognising cues to action }\end{array}$ \\
\hline 4 & Social support and motivation & $\begin{array}{l}\text { Social support and motivation, and } \\
\text { self-monitoring with others }\end{array}$ & $\begin{array}{l}\text { Social support and motivation, self-monitoring } \\
\text { and forming activity routines with others }\end{array}$ \\
\hline 5 & $\begin{array}{l}\text { Experiencing barriers to being } \\
\text { active and staying motivated }\end{array}$ & $\begin{array}{l}\text { Experiencing barriers to being active } \\
\text { and staying motivated; relapse prevention }\end{array}$ & $\begin{array}{l}\text { Experiencing barriers to being active and staying } \\
\text { motivated; reasons for falling back into bad habits; } \\
\text { relapse prevention }\end{array}$ \\
\hline 6 & Summary: staying motivated & $\begin{array}{l}\text { Summary: staying motivated and } \\
\text { self-regulating }\end{array}$ & $\begin{array}{l}\text { Summary: staying motivated, self-regulating and } \\
\text { maintaining habits }\end{array}$ \\
\hline
\end{tabular}

notification asking users to use a star rating system (What did you think of this feedback? 0 to 5 stars) and a qualitative feedback box to receive open-ended comments relating to each session content (Why did you rate it this way? [optional]).

During each intervention session, participants will receive personally-relevant content incorporating $\mathrm{BCTs}$ relevant to their allocated intervention condition (Table 2). To generate the content-specific information, participants will be asked about their physical activity during the previous week at the beginning of each intervention session (including their low, moderate and high intensity physical activity, and resistance training, specifying number and length of sessions). They will also be asked questions regarding the psychological determinants of physical activity, physical resources, and social and environmental factors relevant to the condition to which they are allocated. For example, during the session on goal setting, participants allocated to the Motivation condition will receive information which prompts them to form activity goals and to stay motivated to achieve them. Participants allocated to the Self-regulation condition will also receive suggestions how to monitor and self-regulate towards the goal (action planning, coping planning). Participants assigned to the Habit condition will also receive information on the importance of repetition of the same activity in the same context to develop habits.

The platform uses if-then algorithms to provide tailored feedback based on the participant's responses. The feedback will be drawn from a database of messages incorporating feedback combinations tailored to the participant's condition and responses to prompts for information on the website. The platform will store participants' responses for each session, permitting tailoring of the intervention to the responses from the current session, as well as to responses from previously completed surveys. For instance, graphs displayed to the participants will include changes in physical activity throughout the intervention.
In addition, all groups, with the exception of the control group, will receive weekly text messages sent at the same time each week to provide further condition- and session-relevant feedback based on their most recently completed session and including a short summary of key points. There will be three introduction messages and each session has two session specific text messages, followed by a final concluding message. Participants will also receive regular fortnightly reminders to login to the intervention website when a new session is available sent via email. Up to three reminders will be sent per session (one every four days).

\section{Measures}

Table 3 describes measures that will be taken during the study, specifying the type of outcome assessed, the measurement tool used, the number of questions included, and the time points when the measures will be administered.

\section{Physical activity}

The primary outcome will be measured physical activity over a one-week period prior to the intervention (Time 1) and for a further week immediately post-intervention at the three-month follow-up occasion (Time 2) using accelerometers (GENEActiv Ltd.) [47, 67]. Participants will be asked to wear the unit continuously for the seven day period on the non-dominant upper arm to comply with hospital hygiene regulations. The GENEActiv device is water proof and participants will be informed that they are free to choose whether to wear it while swimming, showering, or sleeping. Data will be collected at $60 \mathrm{~Hz}$ epoch frequency. Step counts will be derived from the acceleration data using open-source macros and they will be used as proxy for physical activity [47]. Participants will be supplied with a band specifically designed to fasten the monitor to the upper arm. 


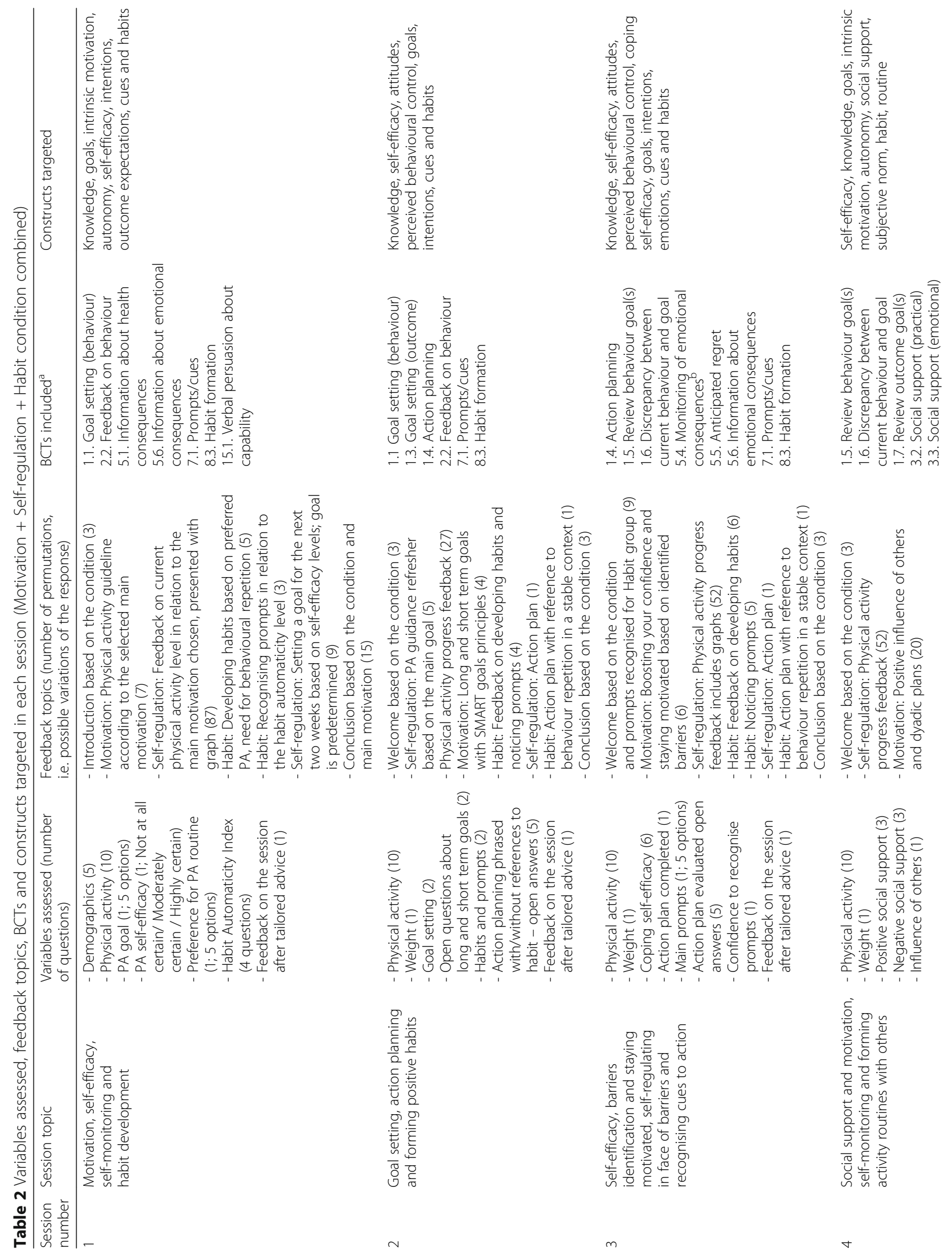


Kwasnicka et al. BMC Public Health (2017) 17:518

Page 10 of 16

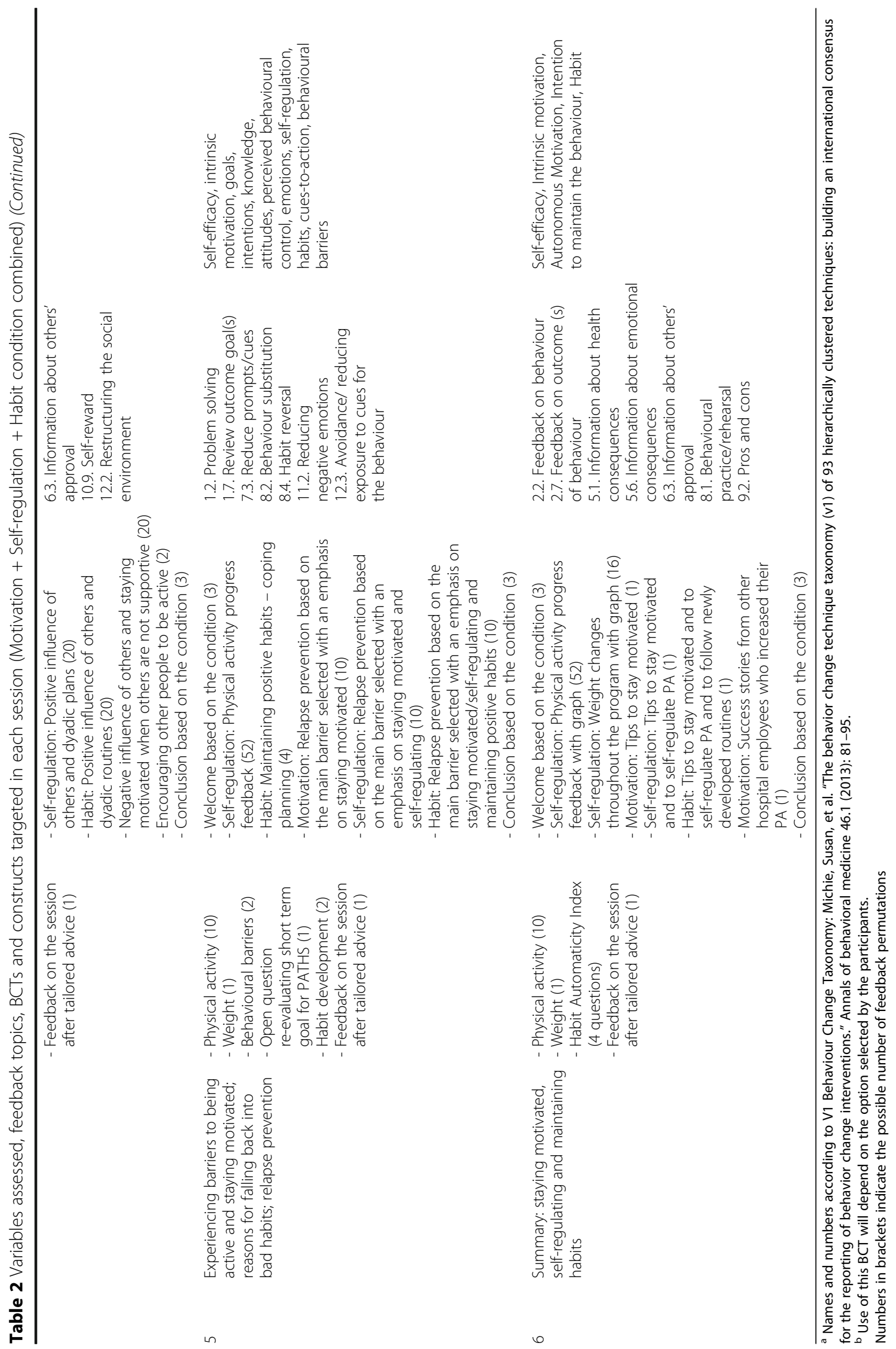


Table 3 Measures taken at Time 1 and Time 2 and outcomes assessed

\begin{tabular}{|c|c|c|c|c|}
\hline \multirow[t]{3}{*}{ Measurement tool } & \multirow[t]{3}{*}{ Reference } & \multicolumn{2}{|c|}{ Number of items } & \multirow[t]{3}{*}{ Outcome } \\
\hline & & Time 1 & Time 2 & \\
\hline & & (baseline) & (3 months) & \\
\hline \multicolumn{5}{|l|}{ Survey measures } \\
\hline $\begin{array}{l}\text { Confirmation of eligibility to } \\
\text { participate in the study }\end{array}$ & N/A & 8 & - & Eligibility \\
\hline $\begin{array}{l}\text { Physical Activity Readiness } \\
\text { Questionnaire (PAR-Q) }\end{array}$ & {$[45]$} & 8 & - & Readiness to undertake PA \\
\hline Demographics & Commonly used items & 14 & 6 & $\begin{array}{l}\text { Age, sex, marital status, ethnicity, } \\
\text { education, weight, height, house income, postcode }\end{array}$ \\
\hline $\begin{array}{l}\text { International Physical Activity } \\
\text { Questionnaire (short version) }\end{array}$ & {$[68,69]$} & 7 & 7 & Physical Activity \\
\hline Job-related physical activity & $\begin{array}{l}\text { From: International Physical } \\
\text { Activity Questionnaire (long } \\
\text { version) [68] }\end{array}$ & 8 & 8 & Job-related physical activity \\
\hline $\begin{array}{l}\text { The Pittsburgh Sleep Quality } \\
\text { Index (PSQI) }\end{array}$ & [70] & 9 & 9 & Sleep \\
\hline $\begin{array}{l}\text { Theory-relevant determinants } \\
\text { of physical activity }\end{array}$ & $\begin{array}{l}\text { Individual items taken from } \\
\text { different questionnaires }\end{array}$ & 30 & 30 & $\begin{array}{l}\text { Motivation, attitudes towards regular physical activity, } \\
\text { outcome expectations, perceived behavioural control, } \\
\text { subjective norms, intentions, barriers self-efficacy, action } \\
\text { planning and coping planning, self-efficacy, goal } \\
\text { facilitation and goal conflict }\end{array}$ \\
\hline $\begin{array}{l}\text { Self-report behavioural } \\
\text { automaticity index }\end{array}$ & {$[77]$} & 8 & 8 & Behaviour automaticity \\
\hline $\begin{array}{l}\text { Personal Need for Structure } \\
\text { Scale }\end{array}$ & {$[78]$} & 12 & - & Personal Need for Structure \\
\hline Physical activity intentions & {$[72]$} & 3 & 3 & Physical Activity Intentions \\
\hline $\begin{array}{l}\text { Depression Anxiety Stress } \\
\text { Scale } 21 \text { (DASS 21) }\end{array}$ & {$[79]$} & 21 & 21 & Depression, Anxiety, Stress \\
\hline SF 12 & {$[80]$} & 12 & 12 & Quality of life \\
\hline $\begin{array}{l}\text { Physical Activity } \\
\text { Neighbourhood Environment } \\
\text { Survey (PANES) }\end{array}$ & {$[81]$} & 17 & - & Perceptions of the environment in relation to PA \\
\hline $\begin{array}{l}\text { Internet use and access to } \\
\text { the intervention }\end{array}$ & Items developed for this study & - & 4 & Internet use and access to the intervention \\
\hline Internet self-efficacy scale & {$[83]$} & 9 & - & Internet self-efficacy \\
\hline SUS and satisfaction & {$[82]$} & - & 16 & SUS and satisfaction \\
\hline Physical advice acceptability & {$[66]$} & - & 15 & Physical advice acceptability \\
\hline Delivery mode usability & [66] & - & 5 & Delivery mode usability \\
\hline Usefulness questions & {$[66]$} & - & 5 & Intervention usefulness \\
\hline Format-related questions & {$[66]$} & - & 9 & Format \\
\hline Total items & & 158 & 158 & \\
\hline \multicolumn{5}{|c|}{ Outcome measures and moderators } \\
\hline $\begin{array}{l}\text { Physical activity objectively } \\
\text { measured with GENEActiv }\end{array}$ & {$[47,67]$} & $\checkmark$ & $\checkmark$ & Objectively measured physical activity \\
\hline $\begin{array}{l}\text { Sleep objectively measured } \\
\text { with GENEActiv }\end{array}$ & {$[47]$} & Optional & Optional & Objectively measured sleep \\
\hline Planning skill task & [90] & $\checkmark$ & - & Planning skills/ability \\
\hline
\end{tabular}

Secondary outcomes will be assessed at baseline (Time 1) and post-intervention (Time 2), and will include subjective measures of physical activity, sitting time, sleep, depression, anxiety, stress, quality of life, theory-derived correlates of physical activity and self-reported weight. Demographics, perceived neighbourhood environment, personal need for 
structure, internet self-efficacy, and planning skills/ability will be assessed at baseline (Time 1) only. Secondary outcomes assessed only at the post-intervention stage (Time 2) will include measures of intervention accessibility, usefulness and satisfaction with the intervention, as well as acceptability of the advice provided. Participants' website usage (e.g., time spent on pages with specific intervention content, number of website visits) will be assessed throughout the study using Google Analytics.

\section{Physical activity (self-reported) and sitting time}

Self-reported activity data will be assessed using a validated and reliable International Physical Activity Questionnaire (IPAQ) short version and work-related IPAQ module from the long version $[68,69]$.

\section{Sleep}

Sleep duration and quality will be measured using the valid and reliable Pittsburgh Sleep Quality Index [70]. We will also derive an objective measure of sleep using the GENEActiv activity monitors for study participants who choose to wear them during their sleep [71]. Total sleep time, number of awakenings, sleep onset and offset will be extracted from the device using open source macros [47].

\section{Theory-derived psychological constructs}

Psychological constructs derived from the motivation and volition theories and habit theory will be assessed using previously-validated and reliable psychometric questionnaires adapted to make reference to physical activity. The measures will include self-report measures of intentions [72], motivation, attitudes [73], outcome expectations [74], perceived behavioural control [73], subjective norms [73], barriers self-efficacy [75], action planning and coping planning [76], self-efficacy, goal facilitation and goal conflict [61]. Habit strength will be measured using the self-report behavioural automaticity index $[63,77]$. Individual differences in tendencies to follow routines will be assessed using the personal need for structure scale [78].

\section{Depression, anxiety and stress}

Validated and reliable Depression, Anxiety and Stress Scale (DASS21) will be used to measure negative affective outcomes [79].

\section{Quality of life}

Quality of life will be assessed with the widely used, valid and reliable Medical Outcomes Survey - Short Form 12 (MOS-SF12) measure [80].

\section{Perceived environmental factors}

Participants ratings of the extent to which their home neighbourhood environment is supportive and conducive to physical activity will be assessed with the validated and reliable Physical Activity Neighbourhood Environment Survey (PANES) [81].

\section{Internet use and intervention accessibility}

Internet use and access will be measured with items developed specifically for this study. Usefulness and satisfaction with the intervention will be measured with the System Usability Scale [82]. Participants' confidence in their ability to use the internet will be measured with validated and reliable internet self-efficacy scale [83].

\section{Characteristics of the intervention}

Acceptability of the advice provided, intervention delivery and format will be measured with items used in previous studies [66].

\section{Demographics}

Participants will be prompted to self-report a number of key demographic details: age, sex, marital status, ethnicity, education, weight, height, household income, postcode, the number of hours and days worked per week, work level (e.g., clinical nurse manager, registered nurse, patient care assistant), salary brackets associated with each employment level, type of work undertaken (e.g., shift-worker, non-shift worker, mix), and absenteeism in the last 3 months.

\section{Adverse effects}

Participants will be encouraged to report any adverse effects they may experience during their participation in the intervention over the email or phone call.

\section{Sample size}

Reviews of behavioural intervention studies have reported small to medium effects of web-delivered interventions aimed at increasing physical activity [84, 85]. Studies that excluded participants who are already active, which most closely represent the current protocol, reported a small-to-medium effect $(d=0.28)$ [84]. Therefore, to detect a similar effect on physical activity between intervention and control group, a total of 134 participants will be required to achieve $80 \%$ power with alpha set at $p<.05$. Based on an estimated drop out of $30 \%$ from web-based tailored interventions to increase physical activity [84], the final target sample size of 192 participants (48 per group) will be recruited at baseline. The study is powered to detect differences between the intervention group and control group (i.e., parallel 2-arm trial); not between the four study arms. 


\section{Study hypotheses}

1. We hypothesise significant increases in physical activity between baseline and 3-month follow-up in the three intervention conditions but no change in physical activity levels in the control group

2. We hypothesise that increases in physical activity at 3-month follow-up will be greatest in the habit intervention condition with smaller changes for the self-regulation and motivation conditions.

3. We hypothesise that participants allocated to the habit condition will have maintained the physical activity changes over time relative to the selfregulation, motivation, and control conditions at 3-month follow-up

4. We hypothesise that higher engagement in the program (e.g., frequency of logins, number of sessions completed, reported relevance and usefulness of the sessions content) will lead to greater change in physical activity at 3-month follow-up in all intervention groups.

5. We hypothesise that the effects of the habit condition on physical activity behaviour will be mediated by the self-reported habit development, effects of the self-regulation condition will be mediated by self-efficacy and planning, and the motivation condition will be mediated by the social cognitive variables of attitudes, motivation, and intention.

\section{Study analysis}

The intervention will be evaluated following the recent guidance on process evaluation for complex interventions [86]. Data will be analysed using generalized linear mixed modelling (GLMM) [87] with a 4 (condition: control, motivation, self-regulation, habit) $\times 2$ (time: baseline, 3 -month follow-up) mixed-model design, accounting for nesting within hospital. Models will be conducting to compare change between conditions of the primary outcome (physical activity) and secondary outcomes (e.g., theory-derived psychological constructs, DASS21, MOSSF12, and absenteeism). Mediation analyses will be conducted using path analytic models using Preacher and Hayes' bootstrapped approach for multiple mediation [88]. Separate path models will be conducted for each condition. Each condition will be represented by a dichotomous dummy-coded variable $(0=$ did not receive intervention component, 1 = received intervention component). Each condition variable will be set as a predictor of physical activity at follow up with motivation and intention (motivation condition), perceived behavioural control and planning (self-regulation condition), and self-reported habit (habit condition) as multiple mediators in each analysis, respectively. Baseline physical activity will be included as a control variable. Intention-to-treat (baseline carried forward) will be utilised for missing data from drop-out. Other missing data will be imputed using multiple imputation with chained equations [89]. Both intention-to-treat and completers analyses will be reported.

\section{Discussion}

Participation in regular physical activity shown to be related to improved health outcomes, reduced health care costs, and reduced disease risk (e.g., cardiovascular disease, Type 2 diabetes). Healthcare professionals including midwives, nurses and patient assistants are often involved in providing health-related behaviour change advice, yet their own health behaviour is relatively poor as demonstrated in occupational studies [7-9]. The PATHS study will examine the effectiveness of a webbased intervention evaluating different behavioural strategies to increase physical activity. The primary aim of the current trial is to test the main and additive effects of different theoretically-derived BCTs - motivational, selfregulatory and habit-based strategies - on physical activity and a range of secondary outcomes. We hypothesise that the habit condition, which includes the highest number of $\mathrm{BCTs}$ will be the most effective. However, we also acknowledge the potential for a larger number of BCTs may create problems for messages to be assimilated and recalled due to the relatively large amount of information provided. The greater information load may undermine, or even overturn, the effects of the BCTs on behaviour change. In addition, the current design does not allow for tests of interactions among sets of BCTs, rather the approach is focused on additive effects i.e. whether motivational interventions that include additional components (e.g., BCTs related to habit) are more effective compared to those that do not. However, this does not rule out the possibility of interactions among the different components affecting the result. Research aimed at identifying the optimum number and combination of BCTs is required to inform the design of future complex interventions.

In addition, the current study will focus on testing theoretically-distinct groups of techniques and their additive effects on health behavior change. The findings of the study will enable us to evaluate the potential mechanisms by which BCTs derived from motivation, volition and habit theories affect change in healthrelated behaviour. Our research will also enable us to test the mediators of the intervention effects to increase participants' physical activity and quality of life, and to reduce work-related stress and absenteeism. We will be able to do this by comparing the effects of each intervention component (e.g., self-regulation) on outcomes mediated by specific theory-based psychological constructs (e.g., planning, self-efficacy) conceptually related to the component. 
Personal characteristics of the intervention participants will be assessed to determine for whom the different intervention content is most suitable. Potential moderators of intervention effects will include level of engagement in the program and planning skills. Determining the characteristics of potential users will enable the future matching of participants with the most suitable intervention content. In addition, by providing opportunity for participants to report on the usability of the platform in healthcare professionals, we will be able to ascertain the extent to which the online intervention is feasible in this population and whether it would likely be used by the target population if it were rolled-out on a large scale.

\section{Implications}

The study described here has important implications for public health practitioners and researchers designing behaviour change interventions. The intervention will make a unique contribution to physical activity promotion and health research by testing the effectiveness of a computer-tailored web-based intervention in promoting physical activity and stress reduction in hospital workers; an under-researched group at risk of high stress. In addition, the intervention will add to theory and knowledge on behaviour change in health contexts by testing whether the inclusions of self-regulatory and habitforming BCTs are more effective in promoting behaviour change and health-related outcomes relative to BCTs targeting motivation alone and an information only control. This is important as it will assist in elucidating the 'active ingredients' of the intervention and the mechanisms by which they exert their effects. The current research will also assist in identifying the key mediators of intervention effects, providing data on the mechanisms by which each intervention condition affects changes in physical activity behaviour.

\footnotetext{
Abbreviations

BCT: Behaviour change technique; DASS21: Depression Anxiety and Stress Scale 21; IPAQ: International Physical Activity Questionnaire; PANES: Physical Activity Neighbourhood Environmental Scale; PARQ: Physical Activity Readiness Questionnaire; PATHS: Physical Activity Tailored intervention in Hospital Staff; RCT: Randomised controlled trial; SF12: Short Form 12 (Quality of Life Measure); SMS: Short message service; SUS: The System Usability Scale; TPB: Theory of Planned Behaviour
}

\section{Acknowledgements}

Website software programming was done by Nathaniel Fitzgerald-Hood. The previous version of the physical activity intervention adapted for the purposes of this study was developed by the TaylorActive Team [66]. We would like to acknowledge Site Representatives for each hospital: Professor Yvonne Hauck from King Edward Memorial Hospital; Directors of Clinical Services Karen Gullick and Daniel Heredia from Hollywood Private Hospital; Director of Nursing and Midwifery Laura Colvin and Director of Research Network Nikolajs Zeps from St John of God Subiaco Hospital and Dr Gail Ross-Adjie and Professor Leanne Monterosso from St John of God Murdoch Hospital.

\section{Funding}

This study is funded by Curtin University, Central Queensland University, and a grant from the Health Collaborative Research Network (HCRN) Australia. Vandelanotte (ID100427) and Duncan (ID100029) hold Future Leader Fellowships funded by the National Heart Foundation of Australia and Short (APP1090517) holds a fellowship funded by National Health and Medical Research Council of Australia. Hagger holds a Finnish Distinguished Professor fellowship funded by TEKES, the Finnish funding agency for innovation. We would like to acknowledge additional funding received from St John of God Subiaco Hospital provided to employ a research nurse.

Availability of data and materials

Not applicable.

\section{Authors' contributions}

DK is the lead researcher and project manager. $\mathrm{MH}, \mathrm{CV}$ and $\mathrm{AR}$ are the principal investigators and secured the funding for the study. DK, MH, CV, AR and $B G$ designed $B C T$-cluster specific intervention content and developed project procedures. CV, CS and MD developed the content of the previous version of the physical activity intervention adapted for the purposes of this study. DC is a site investigator, research nurse and public involvement leader. All authors contributed to writing this manuscript and approved its final version.

\section{Competing interests}

The authors have no competing interests to declare.

\section{Consent for publication}

Not applicable.

\section{Ethics approval and consent to participate}

Ethics approval and participants consent were given for this study, ethics committees that approved the study and their approval reference numbers are: Hollywood Private Hospital Ethics Committee Approval: HPH443; King Edward Memorial Hospital Ethics Committee Approval: 2016034EW; St John of God Hospital Ethics Committee Approval: 1008; Curtin University Ethics Committee Approval: HR20/2016.

\section{Publisher's Note}

Springer Nature remains neutral with regard to jurisdictional claims in published maps and institutional affiliations.

\section{Author details \\ 'Health Psychology \& Behavioural Medicine, School of Psychology and Speech Pathology, Faculty of Health Sciences, Curtin University, Perth, Australia. ${ }^{2}$ Physical Activity Research Group, School of Health, Medical and Applied Sciences, Central Queensland University, Rockhampton, QLD, Australia. ${ }^{3}$ Department of Psychology, Institute of Psychiatry, Psychology \& Neuroscience (IoPPN), King's College London, London, UK. ${ }^{4}$ Freemasons Foundation Centre for Men's Health, South Australian Health and Medical Research Institute, Faculty of Health Sciences, The University of Adelaide, Adelaide, Australia. ${ }^{5}$ Priority Research Centre for Physical Activity and Nutrition, Faculty of Health and Medicine, School of Medicine \& Public Health, The University of Newcastle, Callaghan, Australia. ${ }^{6}$ St John of God Subiaco Hospital, Perth, Australia. ${ }^{7}$ Faculty of Sport and Health Sciences, University of Jyväkylä, Jyväkylä, Finland.}

Received: 23 September 2016 Accepted: 11 May 2017

Published online: 26 May 2017

\section{References}

1. Das P, Horton R. Rethinking our approach to physical activity. Lancet. 2012; 380(9838):189-90.

2. Kohl HW, et al. The pandemic of physical inactivity: global action for public health. Lancet. 2012;380(9838):294-305.

3. Warburton DE, Nicol CW, Bredin SS. Health benefits of physical activity: the evidence. Can Med Assoc J. 2006:174(6):801-9.

4. Penedo FJ, Dahn JR. Exercise and well-being: a review of mental and physical health benefits associated with physical activity. Curr OpinPsychiatry. 2005;18(2):189-93. 
5. World Health Organization. Global Recommendations on Physical Activity for Health. 2015 [23rd September 2015]; Available from: http://www.who. int/dietphysicalactivity/factsheet_recommendations/en/.

6. Loprinzi PD, Beets MW. Need for increased promotion of physical activity by health care professionals. Prev Med. 2014;69:75-9.

7. Blake $\mathrm{H}$, et al. 'Do as I say, but not as I do': Are next generation nurses role models for health? Perspect Public Health. 2011;131(5):231-9.

8. Malik S, Blake H, Batt M. How healthy are our nurses? New and registered nurses compared. Br J Nurs. 2011;20(8):489-96.

9. Zapka JM, et al. Lifestyle behaviours and weight among hospital-based nurses. J Nurs Manag. 2009;17(7):853-60.

10. Fie S, Norman IJ, While AE. The relationship between physicians' and nurses' personal physical activity habits and their health-promotion practice: A systematic review. Health Educ J. 2012; doi:10.1177/0017896911430763.

11. Chang EM, et al. Role stress in nurses: review of related factors and strategies for moving forward. Nurs Health Sci. 2005;7(1):57-65.

12. Phiri LP, et al. Nurses' lifestyle behaviours, health priorities and barriers to living a healthy lifestyle: a qualitative descriptive study. BMC Nurs. 2014;13(1):38

13. Owens JA. Sleep loss and fatigue in healthcare professionals. J Perinat Neonatal Nurs. 2007;21(2):92-100.

14. Atkinson G, Davenne D. Relationships between sleep, physical activity and human health. Physiol Behav. 2007;90(2):229-35.

15. Lela M, Frantz JM. Physical activity among nurses in Kanombe Military Hospital. Afr J Physiother Rehab Sci. 2012;4(1-2):63-6.

16. Hutchinson $A D$, Wilson C. Improving nutrition and physical activity in the workplace: a meta-analysis of intervention studies. Health Promot Int. 2012;27(2):238-49.

17. Michie $\mathrm{S}$, et al. A refined taxonomy of behaviour change techniques to help people change their physical activity and healthy eating behaviours: the CALO-RE taxonomy. Psychol Health. 2011;26(11):1479-98.

18. Michie $\mathrm{S}$, et al. The behavior change technique taxonomy ( $\mathrm{v} 1$ ) of 93 hierarchically clustered techniques: building an international consensus for the reporting of behavior change interventions. Ann Behav Med. 2013;46(1):81-95.

19. Marcus $B H$, et al. Evaluation of motivationally tailored vs. standard self-help physical activity interventions at the workplace. Am J Health Promot. 1998; 12(4):246-53.

20. Spittaels $H$, et al. Effectiveness of an online computer-tailored physical activity intervention in a real-life setting. Health Educ Res. 2007;22(3):385-96.

21. Abraham C, Graham-Rowe E. Are worksite interventions effective in increasing physical activity? A systematic review and meta-analysis. Health Psychol Rev. 2009;3(1):108-44.

22. Wight $\mathrm{D}$, et al. Six steps in quality intervention development (6SQulD). J Epidemiol Community Health. 2015; doi:10.1136/jech-2015-205952.

23. Noar SM, Benac CN, Harris MS. Does tailoring matter? Meta-analytic review of tailored print health behavior change interventions. Psychol Bull. 2007; 133(4):673.

24. Krebs P, Prochaska JO, Rossi JS. A meta-analysis of computer-tailored interventions for health behavior change. Prev Med. 2010;51(3):214-21.

25. Lustria MLA, et al. Computer-tailored health interventions delivered over the Web: review and analysis of key components. Patient Educ Couns. 2009; 74(2):156-73.

26. Neville LM, O'Hara B, Milat A. Computer-tailored physical activity behavior change interventions targeting adults: a systematic review. Int J Behav Nutr Phys Act. 2009;6(1):30.

27. Kelders SM, et al. Persuasive system design does matter: a systematic review of adherence to web-based interventions. J Med Internet Res. 2012;14(6):e152.

28. Lehto $\mathrm{T}$, Oinas-Kukkonen $\mathrm{H}$. Persuasive features in web-based alcohol and smoking interventions: a systematic review of the literature. J Med Internet Res. 2011;13(3):e46.

29. Petty RE, Cacioppo JT, The elaboration likelihood model of persuasion, in Communication and persuasion. New York: Springer; 1986. p. 1-24.

30. Orr JA, King RJ. Mobile phone SMS messages can enhance healthy behaviour: a meta-analysis of randomised controlled trials. Health Psychol Rev. 2015;9(4):397-416.

31. Webb T, et al. Using the internet to promote health behavior change: a systematic review and meta-analysis of the impact of theoretical basis, use of behavior change techniques, and mode of delivery on efficacy. J Med Internet Res. 2010;12(1):e4.

32. Kroeze W, Werkman A, Brug J. A systematic review of randomized trials on the effectiveness of computer-tailored education on physical activity and dietary behaviors. Ann Behav Med. 2006;31(3):205-23.
33. Ajzen I. The theory of planned behavior. Organ Behav Hum Decis Process. 1991:50(2):179-211.

34. Schwarzer R. Health action process approach (HAPA). Göttingen: Gesundheitspsychologie von A bis Z. Hogrefe; 2002. p. 241-5.

35. Hagger MS, et al. Implementation intention and planning interventions in Health Psychology: Recommendations from the Synergy Expert Group for research and practice. Psychol Health. 2016;31(7):814-39.

36. Bargh J. The four horsemen of automaticity: Intention, awareness, efficiency, and control as separate issues. In: Wyer RS, Srull TK, editors. Handbook of social cognition. New York: Psychology Press; 1994. p. 1-40.

37. Chaiken S, Trope Y. Dual-process theories in social psychology. New York: Guilford Press; 1999.

38. Evans JSB, Frankish KE. In two minds: Dual processes and beyond. Oxford: Oxford University Press; 2009

39. Rebar AL, Dimmock JA, Jackson B, Rhodes RE, Kates A, Starling J, Vandelanotte C A systematic review of the effects of non-conscious regulatory processes in physical activity. Health Psychol Rev. 2016;10(4):395-407.

40. Lally P, Gardner B. Promoting habit formation. Health Psychol Rev. 2013; 7(sup 1):S137-58.

41. Rebar AL, Loftus AM, Hagger MS. Cognitive control and the non-conscious regulation of health behavior. Front Hum Neurosci. 2015;9:122.

42. Gardner, B., A review and analysis of the use of 'habit'in understanding, predicting and influencing health-related behaviour. Health Psychol Rev. 2014 (ahead-of-print);1-19.

43. Verplanken B, Van Knippenberg A. Predicting behavior from actions in the past: Repeated decision making or a matter of habit. J Appl Soc Psychol. 1998;28(15):1355-1.

44. Michie S, et al. From theory-inspired to theory-based interventions: A protocol for developing and testing a methodology for linking behaviour change techniques to theoretical mechanisms of action. Ann Behav Med. 2016;1-12. http://link.springer.com/article/10.1007/s12160016-9816-6.

45. Thomas S, Reading J, Shephard RJ. Revision of the physical activity readiness questionnaire (PAR-Q). Can J Sport Sci. 1992;17(4):338-45.

46. Torgerson DJ. Contamination in trials: is cluster randomisation the answer? Brit Med J. 2001:322:355-7.

47. GENEActiv Ltd. GENEActiv Website. 2016 [cited 2016 10th August 2016]; Available from: http://www.geneactiv.org/.

48. Sabia S, et al. Association between questionnaire-and accelerometerassessed physical activity: the role of sociodemographic factors. Am J Epidemiol. 2014;179(6):781-90.

49. Jones CJ, Smith H, Llewellyn C. Evaluating the effectiveness of health belief model interventions in improving adherence: a systematic review. Health Psychol Rev. 2014;8(3):253-69.

50. Cohen S, Underwood LG, Gottlieb BH (Eds). Social support measurement and intervention: A guide for health and social scientists. Oxford: University Press; 2000.

51. Hill JO, Peters JC. Environmental contributions to the obesity epidemic. Science. 1998:280(5368):1371-4.

52. Gross JJ, Muñoz RF. Emotion regulation and mental health. Clin Psychol Sci Pract. 1995;2(2):151-64.

53. Ashford S, Edmunds J, French DP. What is the best way to change selfefficacy to promote lifestyle and recreational physical activity? A systematic review with meta-analysis. Br J Health Psychol. 2010;15(2):265-88.

54. Biddle S, Soos I, Chatzisarantis N. Predicting physical activity intentions using goal perspectives and self-determination theory approaches. Eur Psychol. 1999;4(2):83.

55. Hagger MS, et al. The processes by which perceived autonomy support in physical education promotes leisure-time physical activity intentions and behavior: a trans-contextual model. J Educ Psychol. 2003;95(4):784.

56. Locke EA, Latham GP. A theory of goal setting \& task performance. New Jersey: Prentice-Hall, Inc; 1990.

57. Hagger MS, Luszczynska A. Implementation intention and action planning interventions in health contexts: State of the research and proposals for the way forward. Appl Psychol Health Well-Being. 2014;6(1):1-47.

58. Marlatt GA, George WH. Relapse prevention: Introduction and overview of the model. Br J Addict. 1984;79(3):261-73.

59. Hagger MS, et al. Ego depletion and the strength model of self-control: a meta-analysis. Psychol Bull. 2010;136(4):495.

60. Baumeister RF, et al. Ego depletion: is the active self a limited resource? J Pers Soc Psychol. 1998;74(5):1252. 
61. Presseau J, et al. Multiple goals and time constraints: perceived impact on physicians' performance of evidencebased behaviours. Implement Sci. 2009;4(77):77

62. Gollwitzer PM. Implementation intentions: strong effects of simple plans. Am Psychol. 1999:54(7):493

63. Phillips LA, Gardner B. Habitual exercise instigation (vs. execution) predicts healthy adults' exercise frequency. Health Psychol. 2016;35(1):69-77.

64. PATHS Study Team. PATHS Intervention Website. 2016 [cited 2016 10th August 2016]; Available from: http://www.paths.org.au/.

65. Dombrowski SU, et al. Identifying active ingredients in complex behavioural interventions for obese adults with obesity-related co-morbidities or additional risk factors for co-morbidities: a systematic review. Health Psycho Rev. 2012;6(1):7-32.

66. Vandelanotte $C$, et al. TaylorActive-Examining the effectiveness of webbased personally-tailored videos to increase physical activity: a randomised controlled trial protocol. BMC Public Health. 2015;15(1):1020.

67. Pavey TG, Gomersall SR, Clark BK, Brown WJ. The validity of the GENEActiv wrist-worn accelerometer for measuring adult sedentary time in free living. J Sci Med Sport. 2016;19(5):395-9.

68. Hagströmer M, Oja P, Sjöström M. The International Physical Activity Questionnaire (IPAQ): a study of concurrent and construct validity. Public Health Nutr. 2006;9(06):755-62.

69. Booth $\mathrm{ML}$, et al. International physical activity questionnaire: 12-country reliability and validity. Med Sci Sports Exerc. 2003;195(9131/03):3508-1381.

70. Buysse DJ, et al. The Pittsburgh Sleep Quality Index: a new instrument for psychiatric practice and research. Psychiatry Res. 1989;28(2):193-213.

71. van Hees VT, et al. A novel, open access method to assess sleep duration using a wrist-worn accelerometer. PLoS One. 2015;10(11):e0142533.

72. Courneya KS, McAuley E. Factors affecting the intention-physical activity relationship: Intention versus expectation and scale correspondence. Res Q Exerc Sport. 1994;65(3):280-5.

73. Rhodes RE, Hunt Matheson D, Mark R. Evaluation of social cognitive scaling response options in the physical activity domain. Meas Phys Educ Exerc Sci. 2010;14(3):137-50.

74. Plotnikoff RC, et al. Validation of the decisional balance scales in the exercise domain from the transtheoretical model: A longitudinal test. Meas Phys Educ Exerc Sci. 2001;5(4):191-206.

75. Marcus BH, et al. Self-efficacy and the stages of exercise behavior change. Res Q Exerc Sport. 1992;63(1):60-6.

76. Sniehotta FF, et al. Action planning and coping planning for long-term lifestyle change: theory and assessment. Eur J Soc Psychol. 2005;35(4):565-76.

77. Gardner B, et al. Towards parsimony in habit measurement: Testing the convergent and predictive validity of an automaticity subscale of the Self-Report Habit Index. Int J Behav Nutr Phys Act. 2012;9(1):102.

78. Thompson MM, et al. The personal need for structure and personal fear of invalidity measures: Historical perspectives, current applications, and future directions. In: Cognitive social psychology: The Princeton symposium on the legacy and future of social cognition. Hove: Psychology Press; 2001. p. 19-39.

79. Henry JD, Crawford JR. The short-form version of the Depression Anxiety Stress Scales (DASS-21): Construct validity and normative data in a large non-clinical sample. Br J Clin Psychol. 2005;44(2):227-39.

80. Ware JE Jr, Kosinski M, Keller SD. A 12-Item Short-Form Health Survey: construction of scales and preliminary tests of reliability and validity. Med Care. 1996;34(3):220-33.

81. Sallis JF, et al. Evaluating a brief self-report measure of neighborhood environments for physical activity research and surveillance: Physical Activity Neighborhood Environment Scale (PANES). J Phys Act Health. 2010;7(4):533.

82. Brooke J. SUS-A quick and dirty usability scale. Usability Eval Ind. 1996;189(194):4-7.

83. Torkzadeh G, Van Dyke TP. Development and validation of an Internet selfefficacy scale. Behav Inform Technol. 2001;20(4):275-80.

84. Davies CA, et al. Meta-analysis of internet-delivered interventions to increase physical activity levels. Int J Behav Nutr Phys Act. 2012;9(1):52

85. Vandelanotte $C$, et al. Website-delivered physical activity interventions: A review of the literature. Am J Prev Med. 2007:33(1):54-64

86. Moore GF, et al. Process evaluation of complex interventions: Medical Research Council guidance. BMJ. 2015;350:h1258.

87. Faraway JJ. Extending the linear model with R: generalized linear, mixed effects and nonparametric regression models. Boca Raton: CRC Press; 2016. p. 124

88. Preacher KJ, Hayes AF. SPSS and SAS procedures for estimating indirect effects in simple mediation models. Behav Res Methods Instrum Comput. 2004;36(4):717-31.
89. Buuren S, Groothuis-Oudshoorn K. mice: Multivariate imputation by chained equations in R. J Stat Software. 2011:45(3):1-67.

90. Cambridge Brain Sciences. Spatial Planning Task. 2016 [cited 2016 10th August 2016]; Available from: http://www.cambridgebrainsciences.com/ browse/planning/test/hampshire-tree-task.

91. Schulz KF, Altman DG, Moher D. CONSORT 2010 statement: updated guidelines for reporting parallel group randomised trials. BMC Med. 2010;8(1):18.

\section{Submit your next manuscript to BioMed Central and we will help you at every step:}

- We accept pre-submission inquiries

- Our selector tool helps you to find the most relevant journal

- We provide round the clock customer support

- Convenient online submission

- Thorough peer review

- Inclusion in PubMed and all major indexing services

- Maximum visibility for your research

Submit your manuscript at www.biomedcentral.com/submit
) Biomed Central 